\title{
Recognition of Postures and Freezing of Gait in Parkinson's Disease Patients Using Microsoft Kinect Sensor
}

\author{
A. Amini Maghsoud Bigy ${ }^{1}$ - IEEE EMBS Member, K. Banitsas ${ }^{1}-$ IEEE Member, A. Badii ${ }^{2}$, J. Cosmas ${ }^{1}$ - \\ IEEE Senior Member
}

\begin{abstract}
Freezing of Gait (FOG) is a disabling symptom and movement disorder, typically associated with the latter stages of Parkinson's disease. In this paper, we propose a novel approach for real-time FOG, tremor monitoring and fall detection, consisting of a 3D camera sensor based on the Microsoft Kinect architecture. The system is capable of recognizing freezing episodes (FOG) in a standstill state, tremors and fall incidents, commonly seen in Parkinson's disease patients. In case of an incident, it automatically alerts relatives and healthcare providers. The system was tested on seven simulated subjects in 12 events indicating that the design was able to detect $99 \%$ of the falling incidents, $91 \%$ of tremor and $92 \%$ of the freezing of gait episodes with an average latency of 300 milliseconds. The performance of the system can be further improved with the deployment of the recently released version of Kinect, capable of providing even higher levels of accuracy.
\end{abstract}

\section{INTRODUCTION}

Parkinson's disease is a degenerative neurological condition in which part of the brain becomes progressively incapacitated over time [1] [2] [3]. The brain's nerve cells produce a vital chemical called Dopamine in order to be able to communicate with each other and send signals to their neighboring nerve cells. This helps the brain to perform its important tasks such as controlling movement, motor functions and possibly other functions related to feeling and mood [4]. The lack of Dopamine equilibrium in the brain can lead to brain disease and malfunction. The main clinical Parkinson's disease features related to body movement include tremor, a spontaneous shaking in particular body parts, rigidity manifesting as muscle stiffness and bradykinesia, slow-paced physical movements [1].

\section{BACKGROUND}

\section{A. Freezing of Gait (FOG)}

Freezing of Gait (FOG) is known as one of the most frustrating and least understood symptoms, which usually occurs in the advanced stages of Parkinson's disease [2] [3]. This becomes more aggressive in narrow places, stressful situations and during turning [3] [5]. It often incapacitates the disease sufferers as the consequences of the Parkinson's disease symptoms, especially FOG and falls have a huge

\footnotetext{
${ }^{1}$ Mr Amini Maghsoud Bigy, Dr. Konstantinos Banitsas and Prof. John Cosmas are with the College of Engineering Design and Physical Sciences, Department of Electronics and Computer Engineering, Brunel University London, UK amin.amini, konstantinos.banitsas, john.cosmas, @ brunel.ac.uk).

${ }^{2}$ Prof. Atta Badii is the Director of the Intelligent Systems Research Laboratory, at the School of Systems Engineering, University of Reading (atta.badii@ reading.ac.uk)
}

impact both on the physical and psychosocial level [2]. Consequently, the patient's quality of life suffers and the healthcare and treatment expenditures as well as the cost of the resulting injuries increase substantially. Recent research [6] has shown that about $30 \%$ of Parkinson's disease sufferers experience a sudden freeze (FOG) whereupon patients muscles literally freeze in place as they attempt to walk. Random shaking (also known as Tremor) is another physical symptom of Parkinson's disease. It is usually accompanied by rigidity (stiffness) and slowness of movement, although not every patient diagnosed with Parkinson's disease experiences such symptoms.

\section{B. The Kinect Technology}

The Kinect sensor is a motion-sensing device that enables users to interact with video games without the need for a conventional controller. It consists of two cameras/sensors including a color sensor and an infrared (IR) depth sensor that receives and interprets IR signals, allowing it to work in the dark. There is also an IR emitter, which casts IR light upon the object that can be received by the IR depth sensor in order to understand and map a 3D space. Microsoft has made the Kinect sensor available beyond the Xbox 360 console to home computers with a dedicated Software Development Kit (Kinect SDK) and related documentations [7]. This has enabled developers to take advantage of the Kinect sensor hardware capabilities, creating a plethora of innovative applications; many related to medicine and biomedical engineering.

\section{State of the Art}

Since the release of Kinect SDK, many attempts have been made to use of the Kinect sensor for Parkinson's disease related research. Most of the projects have focused on rehabilitation purposes and experimental ways of monitoring patients' activities.

In [5], a home tracking system available by using Microsoft Kinect sensors was developed to help reduce the incidence of FOG episodes for patients diagnosed with Parkinson's disease. They interconnected several Kinect sensors together in order to have full coverage of the testing environment. Their model operated independently of the cameras and collected data from all the sensors, storing them into a centralized database for further analysis and processing. They used a model based on the subject's histogram of color and height, together with the known average movement delays between each camera. 
In another attempt, Kathryn LaBelle [8] evaluated software that utilized the Microsoft Kinect to aid therapies for stroke survivors in order to help them restore balance and re-gain their previous degree of mobility. It was concluded that the results were promising especially in joint operation monitoring and movement tracking.

Two independent research projects [9] [10] have been carried out for real-time fall detection. Both used the same methodology as deployed in this paper by taking into account the velocity and inactivity patterns to minimize the false positives.

In 2012, a team of researchers and developers known as Team May 1304 [11] created a software system called Project Theia. The purpose of this project was to monitor and track patients' therapy management using the Microsoft Kinect sensor. The software motivated Parkinson's sufferers to move their body muscles by playing a game, and music was part of that therapy. The software was in fact a game that ran on the patient's computer and the information about the game progress could be viewed via a web portal.

To our knowledge, there has been no other substantial research towards real-time, non-invasive detection and recognition of patients with Parkinson's disease symptoms, especially for FOG/tremor incidents. Most of the researchers have concentrated on helping the already diagnosed patients have a better quality life. They have focused on the rehabilitation process by developing games or monitoring systems. Studies such as [12] [13] used a device or a sensor to be attached to or worn by the patients in order to detect the symptoms.

The proposed project is concerned with the deployment of the Microsoft Kinect, as a mainstream sensor to detect and recognize Parkinson's disease postures so that the early symptoms can be detected and categorized, allowing the patient to be monitored and helped at the early stages of the disease. Furthermore, the open design of our system supports connectivity to external devices (e.g. projectors, media players) that can project visual and play auditory cues in an attempt to "unfreeze" [14] [15].

\section{SYSTEM ARCHITECTURE}

The software was written in Microsoft Visual Studio 2013 Professional environment using Visual C\# language.

\section{A. Fall Detection Algorithm}

The falling detection algorithm is designed in order to track the subject's head at all times. Once a fall incident takes place, the algorithm is able to detect it so that the system can respond accordingly. Near miss incidents are not tracked in the current implementation, as the focus of this project was to detect hazardous falls. Equation 1 shows the formula used in the implementation of the fall detection algorithm.

$$
\text { Falling }(f)=\{\forall(\alpha, a) \in X \mid X \subset(-\infty,-1]\}, t=10 \text { min }
$$

Equation 1. Fall detection algorithm

This states that if $\alpha<=-1$ and a $<=-1$ for more than 10 minutes, then a fall incident has taken place. ' $\alpha$ ' is the former head position (Y) axis and ' $a$ ' is the latter head position (Y) axis. In this example, the value of 10 minutes was used to minimize false positives although this can be user defined. The system is able to distinguish the real incident from false positive situations such as when the subject suddenly leaves the scene or bends over. This is accomplished by taking into account the head's velocity and acceleration.

In order for the system to determine whether the subject has recovered his/her posture after a fall incident, the following equation was used.

$$
\begin{gathered}
\text { Standing }(\mathbf{s})=\forall\{\mathbf{a} \in(-1,+\infty) \& \alpha \in(-\infty,-1]\} \text { or } \forall\{\mathbf{a} \in(- \\
\quad 1,+\infty) \& \alpha \in(-\infty,-1]\} \\
\text { Equation 2. Standing detection algorithm }
\end{gathered}
$$

In this instance, if ( $\mathrm{a}>-1$ and $\alpha<=-1$ ) or ( $\mathrm{a}>-1$ and $\alpha>-$ 1 ), then the subject has recovered to a standing position following a fall incident. Where ' $\alpha$ ' is the former head position ( $\mathrm{Y}$ ) axis and ' $\mathrm{a}$ ' is the latter head position $(\mathrm{Y})$ axis.

\section{B. FOG/Tremor Algorithm}

A rather innovative method was applied for the tremor incident detection. The patient's position recognition technique (Figure 2), alongside two body joints (left and right hands) and four variables (two for each joint) were correlated to arrive at the decision regarding the FOG/tremor detection. For tremor detection, the following equation was used:

\section{Tremor $=\forall\{\gamma \in(1,3]$ or $\delta \in(1,3]\} \& \forall\{\varepsilon \in(1,3] \& \zeta$ $\in(1,3]\}$}

Equation 3. Tremor detection algorithm

In this equation, if $((0<\gamma<=3 \mathrm{~cm}$ or $1 \mathrm{~cm}<\delta<=3 \mathrm{~cm})$ and $(0<\varepsilon<=3 \mathrm{~cm}$ or $1<\zeta<=3 \mathrm{~cm}))$, then a tremor episode has occurred. Whereby:

' $\gamma$ ' is (the latter right hand position (X) axis - the former right hand position (X) axis)

' $\delta$ ' is (the former right hand position (X) axis - the latter right hand position (X) axis)

' $\varepsilon$ ' is (the latter left hand position $(\mathrm{X})$ axis - the former left hand position $(\mathrm{X})$ axis)

' $\zeta$ ' is (the former left hand position $(\mathrm{X})$ axis - the latter left hand position $(\mathrm{X})$ axis).

The values one and three in the above equation for both $\gamma$ and $\delta$ were determined by considering an average hand tremor movement in the $\mathrm{X}$ angle.

\section{Algorithm Constant}

For all of the above algorithms, two values for each tracking joint have been recorded with delay latency of $300 \mathrm{~ms}$. For the tremor detection algorithm, it was found that a value between 180 and 320 milliseconds (representing the time delay between each hand's former and latter movements) produced the most accurate results. Finally, it was concluded that the value differences between the realtime (latter) data and the delayed (former) data for each hand should be between zero and four Kinect Depth Measurement (KDM) units; a pre-set value for the hardware to measure 
incidents. Through experimentation, a value of three KDM showed an optimal accuracy.

\section{EXPERIMENT METHODS}

\section{A. Testing Environment}

An initial testing environment (Figure 1) was used to carry out the experiments.

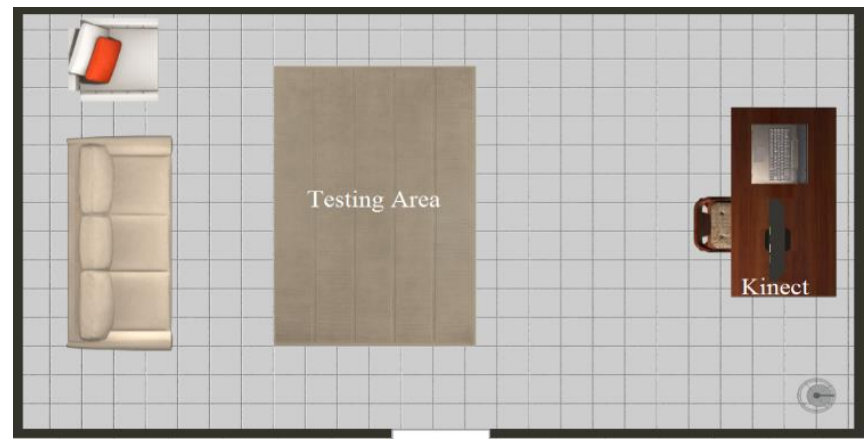

Figure 1. The testing environment

The Kinect sensor was located at a height of approximately 2.2 meters above the floor facing downward and the subject average distance was about 2 meters from the Kinect sensor.

\section{B. Test Cases}

The joint coordination data were gathered from seven subjects (four males and three females aged between 21-32 years old) with different heights, body types, and walking styles. In this first set of experiments, for safety and ethical protection reasons, healthy subjects simulated freezing, tremor and fall incidents (following an ethical approval, real patients will test the system during the next stage). The subjects characteristics used in this project were as follows:

TABLE 1 . Test subjects characteristics ( $n=7 ; 4$ males, 3 females)

\begin{tabular}{|c|c|}
\hline Subject Characteristics & Mean (SD) \\
\hline Age & $27.57(3.95)$ \\
\hline Height (cm) & $175.14(9.87)$ \\
\hline Weight (kg) & $78.42(16.89)$ \\
\hline BMI (kg/m $\left.{ }^{2}\right)$ & $25.3(3.63)$ \\
\hline
\end{tabular}

\section{Results}

Figure 2 shows a graph of the subject's head joint vertical $(\mathrm{Y})$ coordinate in different positions and phases in a given time period with respect to the sensors line of sight. The sensor's orientation and tilt should be considered before initializing the system. The superimposed line chart indicated that the system was able to determine different subject's spatio-temporal position states even during unfavorable lighting conditions.

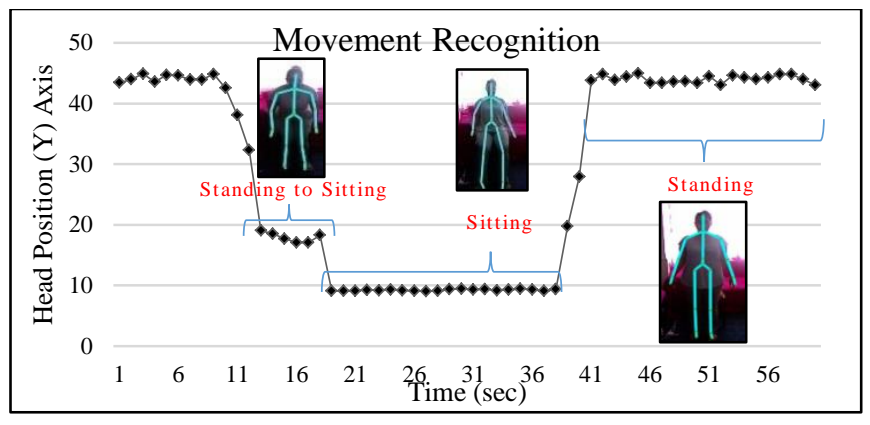

Figure 2. System's movement detection

As expected, joint tracking and skeleton recognition accuracy decreased as the subject's distance from the sensor increased. The analysis showed that the system successfully tracked all the joints and recognized skeletons for distances between one and three meters from the sensor; this is a typical operating range for an average-sized room. The quality of joint tracking was evaluated by placing the subject at different distances in a range of one to four meters. It was decided that the inconsistency of the joint positions increased when the subject was too close or too far away from the sensor. There was a drop in the accuracy at a distance of four meters, where only head and shoulders were recognized correctly and only some of the time. The system was still able to track the joints, although not as accurate as previously.

Figure 3 demonstrates the capability of the system to detect fall incidents by using the fall detection algorithm in Equation 1. During the testing stage, a person simulated falling/standing up at 63 and 105 seconds where our system accurately detected the incidents (the 10-minute delay for falling has been removed in this figure). In our experiments the system was able to recognize the subject's fall with a 99\% overall accuracy. This fall detection performance is achieveable for any subject above 1.5 meters of height (all seven subjects in our experiments). This arises from the fact that it exploits the Kinect skeleton feature [4], and due to the elimination of false positives by taking into account the velocity and inactivity patterns in a testing environment in which no obstacles were involved. After such a detected incident, the system can communicate via an email address and report the incident, including the snapshot of the subject and a time and date stamp. The next step is to conduct the experiment in a real environment with several obsticles and evaluate the detection algorithm.

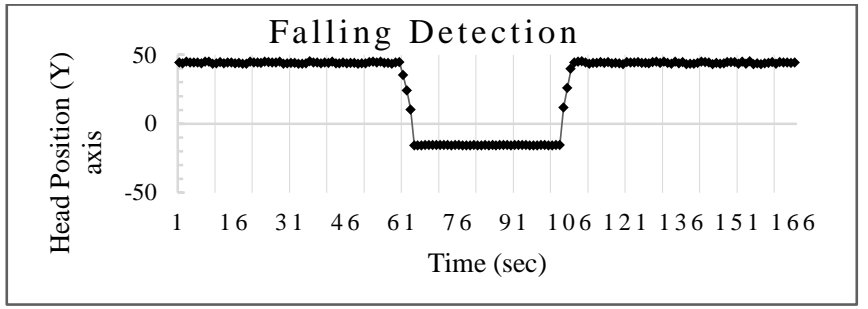

Figure 3. System's falling detection

For the tremor during a FOG event, a probability chart (Figure 4) was created in which the value ' 1 ' indicated the tremor incident and the value ' 0 ' indicated the otherwise. The system showed a $91 \%$ accuracy in detecting tremor episodes; a result adversely impacted by the current 
limitations of the Kinect system, especially due to jittering and low-resolution capabilities. The system achieved a $92 \%$ accuracy in detecting FOG episodes in standstill position with an average latency of 300 milliseconds.

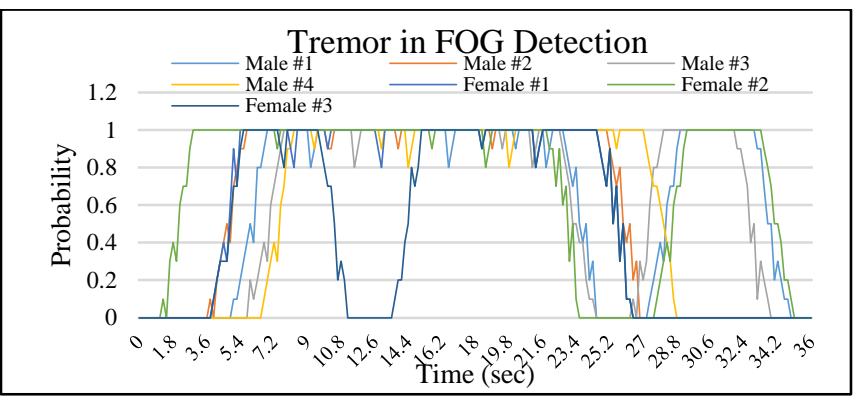

Figure 4. Tremor detection probability

In Figure 5 (left), the application recognized the fall incident according to the subject's position (red circle). Figure 5 (right) illustrates the ability of the system to indicate the tremor episode based on the subject's behaviour. The red-circled area changes to indicate the incident.

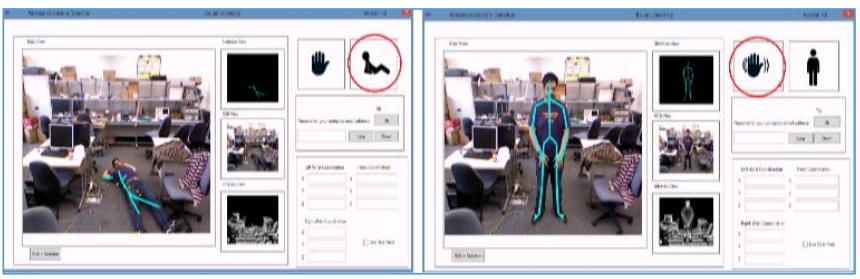

Figure 5. User interface falling/Gait \& tremor notification

\section{CONCLUSION}

During this first phase of our studies on the use of the Kinect sensor as a tool for recognizing Parkinson's disease postures, we concluded that the capabilities and features of the device are well suited to such application domains as investigated in this project. Conclusively, the system presented here is potentially an accurate and valuable tool to recognize FOG and falling episodes in Parkinson's disease patients, following its evaluation on real patients. It requires no on-body sensors; it is non-invasive, easy to setup and provides for an affordable solution. As a next step, our research program is motivated to explore a coupling of the output of this system with a small laser projector so as to exploit both visual and auditory cues to assist the unfreezing of a subject when a FOG episode is detected [14] [15]. This will be further supported by the use of the recently announced new version of Kinect with a considerably enhanced performance, which can powerfully support the detection of hand tremors and enable extended range of view. Moreover, a series of experiments in a real environment with obsticles will be conducted where the fall detection algorithm will be evaluated.

\section{REFERENCES}

[1] Alison Yarnall, Neil Archibald, David Burn, "Parkinson's disease," MOVEMENT DISORDERS, vol. 40, no. 10, p. 529-
535,2012

[2] Bastiaan R. Bloem, Jeffrey M. Hausdorff, Jasper E. Visser, Nir Giladi, "Falls and freezing of gait in Parkinson's disease: A review of two interconnected, episodic phenomena," Movement Disorders, vol. 19, no. 8, pp. 871-884, 2004.

[3] Y. Okuma, "Freezing of gait in Parkinson's disease," Journal of Neurology, vol. 253, no. 7, pp. vii27-vii32, 2006.

[4] H. Staff, "Dopamine," WebMD, 3 December 2010. [Online]. Available: http://www.webmd.com/hw-popup/dopamine. [Accessed 23 August 2013].

[5] Boris Takač, Wei Chen, Matthias Rauterberg, "Toward a domestic system to assist people with Parkinson's," 05 June 2013. [Online]. Available: http://spie.org/x94067.xml. [Accessed 08 September 2013].

[6] J. Gilbert, "Laser Pointers May Help Parkinson's Patients," newswise, 21 April 1999. [Online]. Available: http://www.newswise.com/articles/laser-pointers-may-helpparkinsons-patients. [Accessed 01 September 2013].

[7] M. N. Center, "Microsoft Releases Kinect for Windows SDK Beta for Academics and Enthusiasts," Microsoft, 16 June 2011. [Online]. Available: http://www.microsoft.com/enus/news/press/2011/jun11/06-16MSKinectSDKPR.aspx. [Accessed 18 June 2013].

[8] K. LaBelle, "NetScale Laboratory," 2011. [Online]. Available: http://www1bpt.bridgeport.edu/ jelee/courses/CS550_F13/Thesi s/T3.Kinect\%20Joint.pdf. [Accessed 08 June 2013].

[9] Georgios Mastorakis, Dimitrios Makris, "Fall detection system using Kinect's infrared sensor," J Real-Time Image Proc, Springer, 2012.

[10] Caroline Rougier, Edouard Auvinet, Jacqueline Rousseau, "Fall Detection from Depth Map Video Sequences, Toward Useful Services for Elderly and People with Disabilities," Springer, vol. 6719, pp. 121-128, 2011.

[11] D. Starn, "Parkinson's Therapeutic Game," IOWA STATE UNIVERSITY, 10 May 2013. [Online]. Available: http://seniord.ece.iastate.edu/may1304/index.html. [Accessed 03 September 2013].

[12] Bourke, A.K; O'Brien, J.V; Lyons, G.M, "Evaluation of a threshold-based tri-axial accelerometer fall detection algorithm," Elsevier, vol. 26, no. 2, p. 194-199, 2007.

[13] Alwan, M; Alwan, M; Rajendran, P.J; Kell, S; Mack, D; Dalal, S; Wolfe, M; Felder, R, "A Smart and Passive Floor-Vibration Based Fall Detector for Elderly," in IEEE Information and Communication Technologies, Damascus, 2006.

[14] Prokop T, Schubert M, Berger W, "Visual influence on human locomotion Modulation to changes in optic flow," Springer, vol. 114, no. 1, pp. 63-70, 1997.

[15] Bagley, S; Kelly, B; Tunnicliffe, N; Turnbull, G.I; Walker, J.M , "The effect of visual cues on the gait of independently mobile Parkinson's disease patients," Scopus, vol. 77, pp. 415-420, 1991.

[16] J. C. Augusto, M. Huch, A. Kameas, J. Maitland, P. J. McCullagh, J. Roberts, Andrew Sixsmith, Reiner Wichert, "Handbook of Ambient Assisted Living," in Handbook of Ambient Assisted Living: Technology for Healthcare, Rehabilitation and Well-being, Amesterdam, IOS Press BV, 2012, pp. 3-5.

[17] M. N. Center, "'Kinect for Xbox 360' is Official Name of Microsoft's Controller-Free Game Device," 13 June 2010. [Online]. Available: http://www.microsoft.com/enus/news/features/2010/jun10/06-13kinectintroduced.aspx. [Accessed 18 June 2013].

[18] Coste, Christine Azevedo; Sijobert, Benoît; Pissard-Gibollet, Roger; Pasquier, Maud; Espiau, Bernard; Geny, Christian, "Detection of freezing of gait in Parkinson disease: preliminary results," Sensors, vol. 14, no. 4, pp. 6819 - 6827, 2014.

[19] Tripoliti, EE; Fotiadis, DI; Tzallas, AT; Tsipouras, MG; Rigas, G; Bougia, P; Leontiou, M; Konitsiotis, S; Chondrogiorgi, M; Tsouli, S, "Automatic detection of freezing of gait events in patients with Parkinson's disease," Elsevier, vol. 110, no. 1, pp. 12-26, 2013. 